\title{
FREE GROUPS AND AUTOMORPHISM GROUPS OF INFINITE STRUCTURES
}

\author{
PHILIPP LÜCKE ${ }^{1}$ and SAHARON SHELAH ${ }^{2,3}$ \\ ${ }^{1}$ Mathematisches Institut, Rheinische Friedrich-Wilhelms-Universität Bonn, \\ Endenicher Allee 60, 53115 Bonn, Germany; \\ email: pluecke@uni-bonn.de \\ ${ }^{2}$ Einstein Institute of Mathematics, The Hebrew University of Jerusalem, \\ Edmond J. Safra Campus, Givat Ram, Jerusalem 91904, Israel; \\ email: shelah@math.huji.ac.il \\ ${ }^{3}$ Department of Mathematics, Hill Center-Busch Campus, Rutgers, \\ The State University of New Jersey, 110 Frelinghuysen Road, Piscataway, NJ 08854-8019, USA
}

Received 7 February 2013; accepted 18 March 2014

\begin{abstract}
Given a cardinal $\lambda$ with $\lambda=\lambda^{\aleph_{0}}$, we show that there is a field of cardinality $\lambda$ whose automorphism group is a free group of rank $2^{\lambda}$. In the proof of this statement, we develop general techniques that enable us to realize certain groups as the automorphism group of structures of a given cardinality. They allow us to show that analogues of this result hold for free objects in various varieties of groups. For example, the free abelian group of rank $2^{\lambda}$ is the automorphism group of a field of cardinality $\lambda$ whenever $\lambda$ is a cardinal with $\lambda=\lambda^{\aleph_{0}}$. Moreover, we apply these techniques to show that consistently the assumption that $\lambda=\lambda^{\aleph_{0}}$ is not necessary for the existence of a field of cardinality $\lambda$ whose automorphism group is a free group of rank $2^{\lambda}$. Finally, we use them to prove that the existence of a cardinal $\lambda$ of uncountable cofinality with the property that there is no field of cardinality $\lambda$ whose automorphism group is a free group of rank greater than $\lambda$ implies the existence of large cardinals in certain inner models of set theory.
\end{abstract}

2010 Mathematics Subject Classification: primary 03E75, 20E05, 20F29; secondary 03E35.

\section{Introduction}

Given an infinite cardinal $\lambda$, we say that a group $G$ is the automorphism group of a $\lambda$-structure if there is a first-order language $\mathcal{L}$ and an $\mathcal{L}$-structure $\mathcal{M}$ such that the cardinality of the signature of $\mathcal{L}$ and the cardinality of the domain of $\mathcal{M}$ are at most $\lambda$ and the group $\operatorname{Aut}(\mathcal{M})$ consisting of all automorphisms of

(c) The Author(s) 2014. The online version of this article is published within an Open Access environment subject to the conditions of the Creative Commons Attribution licence $<$ http://creativecommons.org/licenses/by/3.0/>. 
$\mathcal{M}$ is isomorphic to $G$. The work of this paper is motivated by questions of the following type: given an abstract group $G$ and an infinite cardinal $\lambda$, is $G$ the automorphism group of a $\lambda$-structure? We start by presenting some known results related to this kind of problem.

Let $G$ be a group that is the automorphism group of a $\lambda$-structure for some infinite cardinal $\lambda$. Then $G$ can be embedded into the group $\operatorname{Sym}(\lambda)$ of all permutations of $\lambda$, and therefore has cardinality at most $2^{\lambda}$. It is shown in [6, Section 5.5] and [11, Section 3] that there is a graph of cardinality $\lambda$ whose automorphism group is isomorphic to $G$. Moreover, the results of [4] and [11] show that $G$ is also isomorphic to the automorphism group of a field of cardinality $\lambda$. Hence we only need to consider finite languages and structures of cardinality $\lambda$ if we want to check whether a given group is the automorphism group of a $\lambda$-structure.

In the other direction, if $\lambda$ is an infinite cardinal and $G$ is a group of cardinality at most $\lambda$, then it is easy to construct a first-order language $\mathcal{L}$ of cardinality $\lambda$ and an $\mathcal{L}$-model $\mathcal{M}$ of cardinality $\lambda$ such that the groups $G$ and $\operatorname{Aut}(\mathcal{M})$ are isomorphic. In particular, every infinite group $G$ is the automorphism group of a $|G|$-structure. In contrast, for every infinite cardinal $\lambda$, there is a group of cardinality $\lambda^{+}$that is not the automorphism group of a $\lambda$-structure. For example, De Bruijn showed in [3, Theorem 5.1] that the group Fin $\left(\lambda^{+}\right)$consisting of all finite permutations of $\lambda^{+}$cannot be embedded into the group $\operatorname{Sym}(\lambda)$. Moreover, Sanerib showed in [14, Theorem 2.2] that the group $\operatorname{Sym}(\lambda)$ has $2^{2^{\lambda}}$-many nonisomorphic subgroups. Since, up to isomorphism, there are only $2^{\lambda}$-many fields of cardinality $\lambda$, this shows that there is a subgroup of $\operatorname{Sym}(\lambda)$ that is not the automorphism group of a $\lambda$-structure.

In this paper, we focus on free groups and the following instances of the above problem.

QUESTION 1.1. Given an infinite cardinal $\lambda$, is there a free group of rank greater than $\lambda$ that is the automorphism group of a $\lambda$-structure?

The above question was first asked by David Evans for the case $\lambda=\aleph_{0}$. Its generalization to uncountable cardinalities is motivated by [10, Theorem 1.14].

In [17], the second author showed that Question 1.1 has a negative answer for $\lambda=\aleph_{0}$.

THEOREM 1.2. A free group of uncountable rank is not the automorphism group of an $\aleph_{0}$-structure. 
The methods developed in the proof of this theorem can be generalized to higher cardinalities to prove the next result that answers Question 1.1 in the negative for singular strong limit cardinals of countable cofinality (see [17, Remark 5.2]).

THEOREM 1.3. Let $\left\langle\lambda_{n} \mid n<\omega\right\rangle$ be a sequence of infinite cardinals with $2^{\lambda_{n}}<$ $2^{\lambda_{n+1}}$ for all $n<\omega$. Define $\lambda=\sum_{n<\omega} \lambda_{n}$ and $\mu=\sum_{n<\omega} 2^{\lambda_{n}}$. Then every free group of rank greater than $\mu$ is not the automorphism group of a $\lambda$-structure.

In contrast, Just, Thomas, and the second author showed in [10, Theorem 1.14] that, given an uncountable cardinal $\lambda$ with $\lambda=\lambda^{<\lambda}$ and a cardinal $\nu>\lambda$, there is a cofinality-preserving forcing extension ofthe ground model that adds no new sequences of ordinals of length less than $\lambda$ and contains a graph of cardinality $\lambda$ whose automorphism group is a free group of rank $v$. This shows that it is consistent with the axioms of set theory that the above question has a positive answer for some uncountable cardinal.

The following main result of this paper shows that the axioms of ZFC already imply a positive answer to the above question for a large class of cardinals of uncountable cofinality.

THEOREM 1.4. Let $\lambda$ be a cardinal with $\lambda=\lambda^{\aleph_{0}}$. Then the free group of rank $2^{\lambda}$ is the automorphism group of a $\lambda$-structure.

In particular, the free group of rank $2^{2^{\aleph_{0}}}$ is always the automorphism group of a $2^{\aleph_{0}}$-structure. Moreover, a combination of the above results allows us to simultaneously answer Question 1.1 for all infinite cardinals under certain cardinal arithmetic assumptions. The following corollary is an example of such an application.

Corollary 1.5. Assume that the Continuum Hypothesis and the Singular Cardinal Hypothesis hold. Then the following statements are equivalent for every infinite cardinal $\lambda$.

(1) Either $\operatorname{cof}(\lambda)>\omega$ or there is a cardinal $\kappa<\lambda$ with $2^{\kappa}>\lambda$.

(2) There is a free group of rank greater than $\lambda$ that is the automorphism group of a $\lambda$-structure.

We outline the proof of Theorem 1.4. In Section 2, we will show that it suffices to construct an inverse system of groups satisfying certain cardinality assumptions whose inverse limit is a free group of large cardinality. We will construct such 
systems of groups assuming the existence of certain inverse systems of sets in Section 3. Finally, we will use the assumption that $\lambda=\lambda^{\aleph_{0}}$ to construct these systems of sets in Section 4.

In the approach sketched above, we develop general techniques to construct structures with prescribed automorphism group. They will allow us to show that analogues of Theorem 1.4 hold for free objects in various varieties of groups. For example, the free abelian group of rank $2^{\lambda}$ is the automorphism group of a $\lambda$ structure whenever $\lambda$ is a cardinal with $\lambda=\lambda^{\aleph_{0}}$. This contrasts a result of Solecki (see [19, Remark 1.6]) who showed that a free abelian group of uncountable rank is not the automorphism group of an $\aleph_{0}$-structure.

In another direction, the methods developed in the proof of Theorem 1.4 also allow us to show that the cardinal arithmetic assumption that $\lambda=\lambda^{N_{0}}$ is consistently not necessary for the existence of a free group of rank $2^{\lambda}$ that is the automorphism group of a $\lambda$-structure. This statement follows directly from the next result. Given a cardinal $\kappa$, we let $\operatorname{Add}(\omega, \kappa)$ denote the forcing that adds $\kappa$-many Cohen reals to the ground model.

THEOREM 1.6. Let $\lambda$ be a cardinal with $\lambda=\lambda^{\aleph_{0}}$, and let $G$ be $\operatorname{Add}(\omega, \kappa)$-generic over the ground model $\mathrm{V}$ for some cardinal $\kappa$. In $\mathrm{V}[G]$, there is a free group of rank greater than or equal to $\left(2^{\lambda}\right)^{\mathrm{V}}$ that is the automorphism group of a $\lambda$ structure.

The above results raise the question whether the existence of a cardinal $\lambda$ of uncountable cofinality with the property that no free group of rank greater than $\lambda$ is the automorphism group of a $\lambda$-structure is even consistent with the axioms of ZFC. Another byproduct of our constructions is the observation that the existence of such a cardinal has consistency strength strictly greater than that of ZFC. This observation is a consequence of the next result.

Remember that a partial order $\mathbb{T}=\left\langle T, \leqslant_{\mathbb{T}}\right\rangle$ is a tree if $\mathbb{T}$ has a unique minimal element and the set $\operatorname{pre}_{\mathbb{T}}(t)=\left\{s \in T \mid s \leqslant_{\mathbb{T}} t, s \neq t\right\}$ iswell-ordered by $\leqslant_{\mathbb{T}}$ for every $t \in T$. Given such a tree $\mathbb{T}$ and $t \in T$, we define $\operatorname{rnk}_{\mathbb{T}}(t)$ to be the order-type of $\left\langle\operatorname{pre}_{\mathbb{T}}(t), \leqslant_{\mathbb{T}}\right\rangle$. We define the height ht $(\mathbb{T})$ of $\mathbb{T}$ to be the least upper bound of the set $\left\{\operatorname{rnk}_{\mathbb{T}}(t) \mid t \in T\right\}$. Finally, a subset $B$ of $T$ is a branch through $\mathbb{T}$ if $B$ is $\leqslant_{\mathbb{T}}$-downwards closed and $B$ is well-ordered by $\leqslant_{\mathbb{T}}$.

THEOREM 1.7. Let $\lambda$ be a cardinal of uncountable cofinality. If there is a tree of cardinality and height $\lambda$ with more than $\lambda$-many branches of order-type $\lambda$, then there is a free group of rank greater than $\lambda$ that is the automorphism group of a $\lambda$-structure. 
By considering the tree $\left\langle\left({ }^{<\lambda} 2\right)^{M}, \subseteq\right\rangle$ for some inner model $M$, the above result directly implies the following corollary.

COROLLARY 1.8. Let $\lambda$ be a cardinal of uncountable cofinality, and let $M$ be an inner model of ZFC. If $\lambda^{+}=\left(\lambda^{+}\right)^{M}$ and $\lambda=\left(2^{<\lambda}\right)^{M}$, then there is a free group of rank greater than $\lambda$ that is the automorphism group of a $\lambda$-structure.

This statement allows us to directly derive large cardinal strength from the nonexistence of certain automorphism groups.

COROLLARY 1.9. Let $\lambda$ be a regular uncountable cardinal such that there is no free group of rank greater than $\lambda$ that is the automorphism group of a $\lambda$-structure. Then $\lambda^{+}$is an inaccessible cardinal in $\mathrm{L}[x]$ for every $x \subseteq \lambda$.

Proof. Assume, towards a contradiction, that $\lambda^{+}$is not an inaccessible cardinal in $\mathrm{L}[x]$ for some $x \subseteq \lambda$. Then there is a subset $y \subseteq \lambda$ with $\lambda^{+}=\left(\lambda^{+}\right)^{\mathrm{L}[y]}$. Since $\left(2^{<\lambda}\right)^{\mathrm{L}[y]}=\lambda$ holds, we can use Corollary 1.8 to derive a contradiction.

Note that Mitchell used an inaccessible cardinal to construct a model of ZFC in which every tree of cardinality $\aleph_{1}$ and height $\omega_{1}$ has at most $\aleph_{1}$-many branches of order-type $\omega_{1}$ (see [2, Section 8] and [13]). This statement is also a consequence of the Proper Forcing Axiom (see [1, Theorem 7.10]).

In the case of singular cardinals of uncountable cofinality, it is possible to use core model theory (see, for example, [15]) to obtain inner models containing much larger large cardinals from the above assumption.

COROLLARY 1.10. Let $\lambda$ be a singular cardinal of uncountable cofinality such that there is no free group of rank greater than $\lambda$ that is the automorphism group of a $\lambda$-structure. Then there is an inner model with a Woodin cardinal.

Proof. Assume, towards a contradiction, that there is no inner model with a Woodin cardinal. Then we can construct the core model $\mathrm{K}$ below one Woodin cardinal (see [9]). It satisfies the Generalized Continuum Hypothesis and has the covering property. In particular, we have that $\lambda^{+}=\left(\lambda^{+}\right)^{\mathrm{K}}$ and $\left(2^{<\lambda}\right)^{\mathrm{K}}=\lambda$. By Corollary 1.8 , this yields a contradiction.

The results of [16, Section 2] show that the nonexistence of trees with the properties listed in Theorem 1.7 at a singular cardinal of uncountable cofinality is equivalent to a PCF-theoretic statement that is not known to be consistent. Related questions can also be found in [18, Chapter II, Section 6]. 


\section{Realizing limits of groups as automorphism groups}

In this section, we show that certain limit objects in the category of groups can be realized as the automorphism group of a structure whose cardinality only depends on the size of the objects appearing in the corresponding limit construction. We will later use this observation to reduce the problem of constructing structures with a prescribed automorphism group to the problem of constructing inverse systems of groups with a prescribed inverse limit.

The results of this section are formulated using the language of category theory. This approach was suggested to us by one of the anonymous referees. It will allow us to apply our results to a great variety of limit constructions in the category of groups. We start by recalling some basic category theoretical notions. Our account of category theory follows [12].

Definition 2.1. Let $F: \mathrm{J} \longrightarrow \mathrm{C}$ be a functor from a set-sized category $\mathrm{J}$ into a category $\mathrm{C}$.

(1) A cone over $F$ is a pair $\left\langle N,\left\langle\psi_{X} \mid X \in \mathrm{Ob}(\mathrm{J})\right\rangle\right\rangle$ such that $N$ is an object in $\mathrm{C}, \psi_{X}: N \longrightarrow F(X)$ is a morphism in $\mathrm{C}$ for every object $X$ in $\mathrm{J}$, and $\psi_{Y}=F(f) \circ \psi_{X}$ holds for every morphism $f: X \longrightarrow Y$ in $\mathrm{J}$.

(2) A limit of $F$ is a cone $\left\langle L,\left\langle\Psi_{X} \mid X \in \mathrm{Ob}(\mathrm{J})\right\rangle\right\rangle$ over $F$ such that, for every cone $\left\langle N,\left\langle\psi_{X} \mid X \in \mathrm{Ob}(\mathrm{J})\right\rangle\right\rangle$ over $F$, there is a unique morphism $u: N \longrightarrow$ $L$ in $\mathrm{C}$ with $\psi_{X}=\Psi_{X} \circ u$ for every $X \in \mathrm{Ob}(\mathrm{J})$.

EXAMPLE 2.2. Limits exist in the category Set of sets. Let $\mathrm{J}$ be a set-sized category, and let $F: \mathrm{J} \longrightarrow$ Set be a functor. Define $A_{F}$ to be the set of all $\left(a_{X}\right)_{X \in \mathrm{Ob}(\mathrm{J})}$ in $\prod_{X \in \mathrm{Ob}(\mathrm{J})} F(X)$ with the property that $a_{Y}=F(f)\left(a_{X}\right)$ holds for every morphism $f: X \longrightarrow Y$ in J. Given an object $X$ in $\mathrm{J}$, we let $\mathrm{p}_{X}$ : $A_{F} \longrightarrow F(X)$ denote the canonical projection. Then it is easy to check that $\left\langle A_{F}\right.$, $\left.\left\langle\mathrm{p}_{X} \mid X \in \mathrm{Ob}(\mathrm{J})\right\rangle\right\rangle$ is a limit of $F$.

EXAMPLE 2.3. Limits exist in the category Grp of groups. If $\mathrm{J}$ is a set-sized category and $F: \mathrm{J} \longrightarrow$ Grp is a functor, then we can obtain a limit of $F$ by considering the subgroup $G_{F}$ of $\prod_{X \in \mathrm{Ob}(\mathrm{J})} F(X)$ consisting of all $\left(g_{X}\right)_{X \in \mathrm{Ob}(\mathrm{J})}$ with $g_{Y}=F(f)\left(g_{X}\right)$ for every morphism $f: X \longrightarrow Y$ in $\mathrm{J}$ together with the canonical projections $\pi_{X}: G_{F} \longrightarrow F(X)$.

The following result shows that the group $G_{F}$ constructed in Example 2.3 is isomorphic to the automorphism group of a structure of small cardinality. 
THEOREM 2.4. Let $\lambda$ be an infinite cardinal, let $\mathrm{J}$ be a set-sized category of cardinality at most $\lambda$, and let $F: \mathrm{J} \longrightarrow$ Grp be a functor with $|F(X)| \leqslant \lambda$ for every $X \in \mathrm{Ob}(\mathrm{J})$. Then the group $G_{F}$ is the automorphism group of a $\lambda$-structure.

Proof. Define $\mathcal{L}_{F}$ to be a first-order language with the following symbols.

(i) A binary relation symbols $\dot{H}_{f}$ for every morphism $f: X \longrightarrow Y$ in J.

(ii) A binary relation symbol $\dot{R}_{g, X}$ for every object $X$ in $\mathrm{J}$ and every $g \in F(X)$. By our assumptions, the signature of $\mathcal{L}_{F}$ has cardinality at most $\lambda$. We let $\mathcal{M}_{F}$ denote the unique $\mathcal{L}_{F}$-structure with the following properties.

(i) The domain of $\mathcal{M}_{F}$ is the set

$$
M_{F}=\{\langle g, X\rangle \mid X \in \mathrm{Ob}(\mathrm{J}), g \in F(X)\} .
$$

(ii) If $f: X \longrightarrow Y$ is a morphism in $\mathrm{J}$, then

$$
\dot{H}_{f}^{\mathcal{M}_{F}}=\{\langle\langle g, X\rangle,\langle F(f)(g), Y\rangle\rangle \mid g \in F(X)\} .
$$

(iii) If $X$ is an object in $\mathrm{J}$ and $g \in F(X)$, then

$$
\dot{R}_{g, X}^{\mathcal{M}_{F}}=\{\langle\langle h, X\rangle,\langle h \cdot g, X\rangle\rangle \mid h \in F(X)\} .
$$

Since our assumptions imply that the set $M_{F}$ has cardinality at most $\lambda$, it suffices to show that the group $\operatorname{Aut}\left(\mathcal{M}_{F}\right)$ is isomorphic to $G_{F}$. We prove a number of claims that will allow us to construct such an isomorphism.

Claim 1. If $\sigma \in \operatorname{Aut}\left(\mathcal{M}_{F}\right), X \in \mathrm{Ob}(\mathrm{J})$, and $g \in F(X)$, then there is an $h_{g, X}^{\sigma} \in$ $F(X)$ with $\sigma(\langle g, X\rangle)=\left\langle h_{g, X}^{\sigma}, X\right\rangle$.

Proof of the Claim. By the definition of $\mathcal{M}_{F}$, we have that

$$
\langle\langle g, X\rangle,\langle g, X\rangle\rangle,\langle\sigma(\langle g, X\rangle), \sigma(\langle g, X\rangle)\rangle \in \dot{H}_{\mathrm{id}_{X}}^{\mathcal{M}_{F}},
$$

and this shows that there is an $h \in F(X)$ with $\sigma(\langle g, X\rangle)=\langle h, X\rangle$.

Claim 2. If $\sigma \in \operatorname{Aut}\left(\mathcal{M}_{F}\right)$ and $X \in \mathrm{Ob}(\mathrm{J})$, then there is a unique $c_{X}^{\sigma} \in F(X)$ with $\sigma(\langle g, X\rangle)=\left\langle c_{X}^{\sigma} \cdot g, X\right\rangle$ for all $g \in F(X)$.

Proof of the Claim. Set $c_{X}^{\sigma}=h_{\mathbf{1}_{F(X)}, X}^{\sigma} \in F(X)$. Given $g \in F(X)$, we have that

$$
\left\langle\left\langle\mathbb{1}_{F(X)}, X\right\rangle,\langle g, X\rangle\right\rangle,\left\langle\left\langle c_{X}^{\sigma}, X\right\rangle,\left\langle h_{g, X}^{\sigma}, X\right\rangle\right\rangle \in \dot{R}_{g, X}^{\mathcal{M}_{F}},
$$

and hence $h_{g, X}^{\sigma}=c_{X}^{\sigma} \cdot g$. This allows us to conclude that $\sigma(\langle g, X\rangle)=\left\langle c_{X}^{\sigma} \cdot g\right.$, $X\rangle$. 
Claim 3. If $\sigma \in \operatorname{Aut}\left(\mathcal{M}_{F}\right)$, then $\left(c_{X}^{\sigma}\right)_{X \in \mathrm{Ob}(\mathrm{J})}$ is an element of $G_{F}$.

Proof of the Claim. Let $f: X \longrightarrow Y$ be a morphism in J. Then

$$
\left\langle\left\langle\mathbb{1}_{F(X)}, X\right\rangle,\left\langle\mathbb{1}_{F(Y)}, Y\right\rangle\right\rangle,\left\langle\left\langle c_{X}^{\sigma}, X\right\rangle,\left\langle c_{Y}^{\sigma}, Y\right\rangle\right\rangle \in \dot{H}_{f}^{\mathcal{M}_{F}},
$$

and hence $F(f)\left(c_{X}^{\sigma}\right)=c_{Y}^{\sigma}$.

Claim 4. The map

$$
\Phi: \operatorname{Aut}\left(\mathcal{M}_{F}\right) \longrightarrow G_{F} ; \sigma \longmapsto\left(c_{X}^{\sigma}\right)_{X \in \mathrm{Ob}(\mathrm{J})}
$$

is an isomorphism of groups.

Proof of the Claim. Given $\sigma_{0}, \sigma_{1} \in \operatorname{Aut}\left(\mathcal{M}_{F}\right)$ and $X \in \mathrm{Ob}(\mathrm{J})$, we have that

$$
\left(\sigma_{1} \circ \sigma_{0}\right)\left(\left\langle\mathbb{1}_{F(X)}, X\right\rangle\right)=\sigma_{1}\left(\left\langle c_{X}^{\sigma_{0}}, X\right\rangle\right)=\left\langle c_{X}^{\sigma_{1}} \cdot c_{X}^{\sigma_{0}}, X\right\rangle,
$$

and therefore $c_{X}^{\sigma_{1} \sigma_{0}}=c_{X}^{\sigma_{1}} \cdot c_{X}^{\sigma_{0}}$. Since $\Phi\left(\mathrm{id}_{\mathcal{M}_{F}}\right)=\mathbb{1}_{G_{F}}$ holds by the definition of the function $\Phi$, this shows that $\Phi$ is a homomorphism of groups.

Fix an element $\left(g_{X}\right)_{X \in \mathrm{Ob}(\mathrm{J})}$ of $G_{F}$. Then the function

$$
\sigma: M_{F} \longrightarrow M_{F} ;\langle h, X\rangle \longmapsto\left\langle g_{X} \cdot h, X\right\rangle
$$

is an automorphism of $\mathcal{M}_{F}$ with $\Phi(\sigma)=\left(g_{X}\right)_{X \in \mathrm{Ob}(\mathrm{J})}$. This shows that $\Phi$ is surjective. By Claim 2, $\Phi$ is also injective.

This completes the proof of the theorem.

\section{Automorphism groups constructed from inverse systems of sets}

This section shows that the existence of certain inverse systems of sets allows us to realize large free groups as the inverse limits of systems of groups of small cardinality. In combination with the results of the last section, this will enable us to show that the existence of such limits yields the existence of a structure whose automorphism group is a large free group. Again, we start by recalling some standard definitions and presenting the relevant examples.

A directed set is a partial order $\mathbb{D}=\left\langle D, \leqslant_{\mathbb{D}}\right\rangle$ with the property that $D \neq \emptyset$ and for all $p, q \in D$ there is an $r \in D$ with $p \leqslant \mathbb{D} r$ and $q \leqslant \mathbb{D} r$. Given a directed set $\mathbb{D}=\left\langle D, \leqslant_{\mathbb{D}}\right\rangle$, we let $\mathrm{J}_{\mathbb{D}}$ denote the category defined by the following clauses.

(i) The elements of $D$ are the objects of $J_{\mathbb{D}}$. 
(ii) If $p \leqslant_{\mathbb{D}} q$, then $\operatorname{Hom}_{\mathrm{J}_{\mathbb{D}}}(q, p)$ consists of a unique element $a_{p, q}$. Otherwise, $\operatorname{Hom}_{\mathbb{D}_{\mathbb{D}}}(q, p)$ is the empty set.

(iii) If $p \leqslant \mathbb{D} q \leqslant \mathbb{D} r$, then $a_{p, r}=a_{p, q} \circ a_{q, r}$.

Let $\mathbb{D}=\left\langle D, \leqslant_{\mathbb{D}}\right\rangle$ be a directed set, let $\mathrm{C}$ be a category, and let $F: \mathrm{J}_{\mathbb{D}} \longrightarrow \mathrm{C}$ be a functor. Then we call the pair $\left\langle\langle F(p) \mid p \in D\rangle,\left\langle F\left(a_{p, q}\right) \mid p \leqslant \mathbb{D} q\right\rangle\right\rangle$ an inverse system in $\mathrm{C}$ over $\mathbb{D}$, and we call a limit of the functor $F$ an inverse limit of this inverse system.

EXAMPLE 3.1. Let $\mathbb{I}=\left\langle\left\langle A_{p} \mid p \in D\right\rangle,\left\langle f_{p, q}: A_{q} \longrightarrow A_{p} \mid p \leqslant \mathbb{D} q\right\rangle\right\rangle$ be an inverse system of sets over a directed set $\mathbb{D}=\left\langle D, \leqslant_{\mathbb{D}}\right\rangle$. Then the inverse limit of $\mathbb{I}$ consists of the set

$$
A_{\mathbb{I}}=\left\{\left(a_{p}\right)_{p \in D} \in \prod_{p \in D} A_{p} \mid f_{p, q}\left(a_{q}\right)=a_{p} \text { for all } p, q \in D \text { with } p \leqslant \mathbb{D} q\right\}
$$

together with the canonical projections $\mathrm{p}_{q}: A_{\mathbb{I}} \longrightarrow A_{q}$.

EXAMPLE 3.2. Let $\lambda$ be an infinite cardinal, and let $[\lambda]^{\aleph_{0}}$ denote the set of all countable subsets of $\lambda$. Given $u, v \in[\lambda]^{\aleph_{0}}$ with $u \subseteq v$, set $A_{u}={ }^{u} 2$, and define $f_{u, v}: A_{v} \longrightarrow A_{u}$ by $f_{u, v}(s)=s \uparrow u$ for all $s \in{ }^{v} 2$. Let

$$
\mathbb{I}_{\lambda}=\left\langle\left\langle A_{u} \mid u \in[\lambda]^{\aleph_{0}}\right\rangle,\left\langle f_{u, v} \mid u, v \in[\lambda]^{\aleph_{0}}, u \subseteq v\right\rangle\right\rangle
$$

denote the resulting inverse system of sets over the directed set $\left\langle[\lambda]^{\aleph_{0}}, \subseteq\right\rangle$.

Then it is easy to see that

$$
b:{ }^{\lambda} 2 \longrightarrow A_{\mathbb{I}_{\lambda}} ; x \longmapsto(x \uparrow u)_{u \in[\lambda]^{\aleph_{0}}}
$$

is a well-defined bijection between the sets ${ }^{\lambda} 2$ and $A_{\mathbb{I}_{\lambda}}$.

EXAMPLE 3.3. Let $\mathbb{T}=\left\langle T, \leqslant_{\mathbb{T}}\right\rangle$ be a tree. Given $\alpha<\operatorname{ht}(\mathbb{T})$, we let $\mathbb{T}(\alpha)$ denote the set of all $t \in T$ with $\operatorname{rnk}_{\mathbb{T}}(t)=\alpha$. If $t \in T$ and $\alpha \leqslant \operatorname{rnk}_{\mathbb{T}}(t)$, then we let $t \uparrow \alpha$ denote the unique element $s \in T$ with $s \leqslant_{\mathbb{T}} t$ and $\operatorname{rnk}_{\mathbb{T}}(s)=\alpha$.

Given $\alpha \leqslant \beta<\operatorname{ht}(\mathbb{T})$, set $A_{\alpha}=\mathbb{T}(\alpha)$, and

$$
f_{\alpha, \beta}: A_{\beta} \longrightarrow A_{\alpha} ; t \longmapsto t \uparrow \alpha
$$

We let

$$
\mathbb{I}_{\mathbb{T}}=\left\langle\left\langle A_{\alpha} \mid \alpha<\operatorname{ht}(\mathbb{T})\right\rangle,\left\langle f_{\alpha, \beta} \mid \alpha \leqslant \beta<\operatorname{ht}(\mathbb{T})\right\rangle\right\rangle
$$

denote the resulting inverse system of sets over the directed set $\langle\mathrm{ht}(\mathbb{T}), \leqslant\rangle$. 
It is easy to see that the induced map

$$
b: A_{\mathbb{I}_{\mathbb{T}}} \longrightarrow[\mathbb{T}] ;\left(a_{\alpha}\right)_{\alpha<\mathrm{ht}(\mathbb{T})} \longmapsto\left\{a_{\alpha} \mid \alpha<\mathrm{ht}(\mathbb{T})\right\}
$$

is a bijection between the inverse limit $A_{\mathbb{I}_{\mathbb{T}}}$ and the set [T] consisting of all branches through $\mathbb{T}$ of order-type ht $(\mathbb{T})$.

We now consider inverse systems in the category of groups.

EXAMPLE 3.4. Let $\mathbb{I}=\left\langle\left\langle G_{p} \mid p \in D\right\rangle,\left\langle h_{p, q}: G_{q} \longrightarrow G_{p} \mid p \leqslant \mathbb{D} q\right\rangle\right\rangle$ be an inverse system of groups over a directed set $\mathbb{D}=\left\langle D, \leqslant_{\mathbb{D}}\right\rangle$.

Then the inverse limit of $\mathbb{I}$ consists of the subgroup

$$
G_{\mathbb{I}}=\left\{\left(g_{p}\right)_{p \in D} \in \prod_{p \in D} G_{p} \mid h_{p, q}\left(g_{q}\right)=g_{p} \text { for all } p, q \in D \text { with } p \leqslant \mathbb{D} q\right\}
$$

of the product $\prod_{p \in D} G_{p}$ together with the canonical projections $\pi_{q}: G_{\mathbb{I}} \longrightarrow G_{q}$.

Let $\mathbb{I}=\left\langle\left\langle A_{p} \mid p \in D\right\rangle,\left\langle f_{p, q} \mid p \leqslant \mathbb{D} q\right\rangle\right\rangle$ be an inverse system of sets over a directed set $\mathbb{D}=\langle D, \leqslant \mathbb{D}\rangle$, and let $F:$ Set $\longrightarrow$ Grp be a functor. Then

$$
\mathbb{I}_{F}=\left\langle\left\langle F\left(A_{p}\right) \mid p \in D\right\rangle,\left\langle F\left(f_{p, q}\right) \mid p, q \in D, p \leqslant \mathbb{D} q\right\rangle\right\rangle
$$

is an inverse system of groups over $\mathbb{D}$, and

$$
u_{\mathbb{I}, F}: F\left(A_{\mathbb{I}}\right) \longrightarrow G_{\mathbb{I}_{F}} ; g \longmapsto\left(F\left(\mathrm{p}_{q}\right)(g)\right)_{q \in D},
$$

is the unique homomorphism $u: F\left(A_{\mathbb{I}}\right) \longrightarrow G_{\mathbb{I}_{F}}$ with $F\left(\mathrm{p}_{q}\right)=\pi_{q} \circ u$ for all $q \in D$.

Let $F r:$ Set $\longrightarrow$ Grp be the functor that sends a set $A$ to the free group with basis $A$. Note that this definition implies that $\operatorname{Fr}(\emptyset)$ is the trivial group.Assume that $\lambda$ is an infinite cardinal, $\mathbb{D}$ is a directed set of cardinality at most $\lambda$, and $\mathbb{I}$ is an inverse limit of sets of cardinality at most $\lambda$ over $\mathbb{D}$. Since $|\operatorname{Fr}(A)| \leqslant$ $|A|+\aleph_{0}$ holds for every set $A$, Theorem 2.4 implies that the group $G_{\mathbb{I}_{F r}}$ is the automorphism group of a $\lambda$-structure. In particular, if the map $u_{\mathbb{I}, F r}$ defined in (6) is an isomorphism, then the free group of rank $\left|A_{\mathbb{I}}\right|$ is the automorphism group of a $\lambda$-structure.

Given a functor $F:$ Set $\longrightarrow$ Grp and an inverse system $\mathbb{I}$ of sets, the above observation shows that it is natural to ask whether the group $F\left(A_{\mathbb{I}}\right)$ can be the inverse limit of $\mathbb{I}_{F}$. In the remainder of this section, we isolate assumptions on the functor $F$ and the underlying directed set $\mathbb{D}$ that lead to a positive answer 
to this question. This will allow us to use the argument sketched above to prove Theorem 1.4.

We start by discussing the desired properties of the functor $F$. The next definition and the category theoretical formulation of the following results were suggested to us by one of the anonymous referees. Given sets $\bar{A} \subseteq A$, we let $i_{\bar{A}, A}: \bar{A} \longrightarrow A$ denote the canonical inclusion map.

DEFINITION 3.5. A functor $F:$ Set $\longrightarrow$ Grp induces a free construction if the following statements hold.

(1) Given a set $A$ and $g \in F(A)$, there is a unique finite subset $A(g)$ of $A$ such that $g \in \operatorname{ran}\left(F\left(i_{A(g), A}\right)\right)$ and $A(g) \subseteq \bar{A}$ for every finite subset $\bar{A}$ of $A$ with $g \in \operatorname{ran}\left(F\left(i_{\bar{A}, A}\right)\right)$.

(2) Given a set $A$, the homomorphism $F\left(i_{\emptyset, A}\right): F(\emptyset) \longrightarrow F(A)$ is injective.

The functor $\mathrm{Fr}:$ Set $\longrightarrow$ Grp defined above obviously satisfies the assumptions of this definition. Moreover, the construction of free objects in all nontrivial varieties of groups can be realized by a functor with these properties. For example, the above statements are satisfied by the functor that sends a set $A$ to the free abelian group with basis $A$ and the functor that sends a set $A$ to the $A$-fold free product of some fixed group $G$.

LEMMA 3.6. Let $F:$ Set $\longrightarrow$ Grp be a functor that induces a free construction. If $\mathbb{I}$ is an inverse system of sets, then the induced map $u_{\mathbb{I}, F}: F\left(A_{\mathbb{I}}\right) \longrightarrow G_{\mathbb{I}_{F}}$ defined in (6) is injective.

Proof. Let $\mathbb{D}=\left\langle D, \leqslant_{\mathbb{D}}\right\rangle$ be a directed set with $\mathbb{I}=\left\langle\left\langle A_{p} \mid p \in D\right\rangle,\left\langle f_{p, q} \mid p \leqslant \mathbb{D} q\right\rangle\right\rangle$. Fix an element $g \in F\left(A_{\mathbb{I}}\right)$ with $u_{\mathbb{I}, F}(g)=\mathbb{1}_{G_{\mathbb{I}} F}$. Then there is a unique minimal finite subset $A_{\mathbb{I}}(g)$ of $A_{\mathbb{I}}$ with $g=F\left(i_{A_{\mathbb{I}}(g), A_{\mathbb{I}}}\right)(\bar{g})$ for some $\bar{g} \in F\left(A_{\mathbb{I}}(g)\right)$.

First, assume that $A_{\mathbb{I}}(g)=\emptyset$. Pick some $q \in D$. Since the canonical projection $\mathrm{p}_{q}: A_{\mathbb{I}} \longrightarrow A_{q}$ satisfies $i_{\emptyset, A_{q}}=\mathrm{p}_{q} \circ i_{\emptyset, A_{\mathbb{I}}}$ and the canonical projection $\pi_{q}$ : $G_{\mathbb{I}_{F}} \longrightarrow F\left(A_{q}\right)$ satisfies $F\left(\mathrm{p}_{q}\right)=\pi_{q} \circ u_{\mathbb{I}, F}$, we have that

$$
F\left(i_{\emptyset, A_{q}}\right)(\bar{g})=\left(F\left(\mathrm{p}_{q}\right) \circ F\left(i_{\emptyset, A_{\mathbb{I}}}\right)\right)(\bar{g})=\left(\pi_{q} \circ u_{\mathbb{I}, F}\right)(g)=\mathbb{1}_{F\left(A_{q}\right)} .
$$

Since our assumption implies that $F\left(i_{\emptyset, A_{q}}\right)$ is an injection, this shows that $\bar{g}=$ $\mathbb{1}_{F\left(A_{\mathbb{I}}(g)\right)}$, and hence $g=\mathbb{1}_{F\left(A_{\mathbb{I}}\right)}$.

Now, assume that there are pairwise different $\left(a_{p}^{1}\right)_{p \in D}, \ldots,\left(a_{p}^{n}\right)_{p \in D} \in A_{\mathbb{I}}$ with $A_{\mathbb{I}}(g)=\left\{\left(a_{p}^{1}\right)_{p \in D}, \ldots,\left(a_{p}^{n}\right)_{p \in D}\right\}$. Since $\mathbb{D}$ is a directed set, there is a $q_{*} \in D$ with $a_{q_{*}}^{i} \neq a_{q_{*}}^{j}$ for all $i<j \leqslant n$. This implies that the map 
$\mathrm{p}_{q_{*}} \circ i_{A_{\mathbb{I}}(g), A_{\mathbb{I}}}: A_{\mathbb{I}}(g) \longrightarrow A_{q_{*}}$ is injective, and hence $F\left(\mathrm{p}_{q_{*}}\right) \circ F\left(i_{A_{\mathbb{I}}(g), A_{\mathbb{I}}}\right)$ is also injective. Since

$$
\left(F\left(\mathrm{p}_{q_{*}}\right) \circ F\left(i_{A_{\mathbb{I}}(g), A_{\mathbb{I}}}\right)\right)(\bar{g})=\left(\pi_{q_{*}} \circ u_{\mathbb{I}, F}\right)(g)=\mathbb{1}_{F\left(A_{q_{*}}\right)},
$$

we can conclude that $\bar{g}=\mathbb{1}_{F\left(A_{\mathbb{I}}(g)\right)}$ and $g=\mathbb{1}_{F\left(A_{\mathbb{I}}\right)}$ also hold in this case.

Next, we discuss the desired properties of the underlying directed set. Given a directed set $\mathbb{D}=\langle D, \leqslant \mathbb{D}\rangle$, we define an infinite game $\mathcal{G}(\mathbb{D})$ of perfect information between Player I and Player II: in the $i$ th round of this game, Player I chooses an element $p_{2 i}$ from $D$ with $p_{k} \leqslant \mathbb{D} p_{2 i}$ for all $k<2 i$, and then Player II chooses an element $p_{2 i+1}$ from $D$ with $p_{2 i} \leqslant \mathbb{D} p_{2 i+1}$. Player I wins a run $\left(p_{i}\right)_{i<\omega}$ of $\mathcal{G}(\mathbb{D})$ if and only if there is a $q \in D$ with $p_{i} \leqslant \mathbb{D} q$ for all $i<\omega$. If Player I does not win a run of $\mathcal{G}(\mathbb{D})$, then Player II wins the run. A similar game can be used to characterize the $\sigma$-distributivity of Boolean algebras (see [7]).

Proposition 3.7. Given a directed set $\mathbb{D}=\langle D, \leqslant \mathbb{D}\rangle$, the following statements are equivalent.

(1) Player I wins every run of $\mathcal{G}(\mathbb{D})$.

(2) If $A \in[D]^{\aleph_{0}}$, then there is a $q \in D$ with $p \leqslant \mathbb{D} q$ for every $p \in A$.

If these equivalent statements hold, then we say that $\mathbb{D}$ is $\sigma$-directed. In the following, we are interested in a weakening of this property that is defined using the notion of winning strategies.

Definition 3.8. Let $\mathbb{D}=\langle D, \leqslant \mathbb{D}\rangle$ be a directed set.

(1) A function $s:{ }^{<\omega} D \longrightarrow D$ is a strategy for Player II in the game $\mathcal{G}(\mathbb{D})$ if $p_{2 i} \leqslant \mathbb{D} s\left(\left\langle p_{0}, \ldots, p_{2 i}\right\rangle\right)$ holds for all $i<\omega$ and $p_{0}, \ldots, p_{2 i} \in D$.

(2) A strategy $s$ for Player II in the game $\mathcal{G}(\mathbb{D})$ is a winning strategy if Player II wins every run $\left(p_{i}\right)_{i<\omega}$ of $\mathcal{G}(\mathbb{D})$ that is played according to $s$, in the sense that $s\left(\left\langle p_{0}, \ldots, p_{2 i}\right\rangle\right)=p_{2 i+1}$ holds for all $i<\omega$.

Clearly, if $\mathbb{D}$ is $\sigma$-directed, then there is no winning strategy for Player II in the game $\mathcal{G}(\mathbb{D})$.

Proposition 3.9. Let $\mathbb{D}=\langle D, \leqslant \mathbb{D}\rangle$ be a directed set, and let $n: D \longrightarrow \omega$ be a function with $n(p) \leqslant n(q)$ for all $p, q \in D$ with $p \leqslant \mathbb{D} q$. If Player II has no winning strategy in the game $\mathcal{G}(\mathbb{D})$, then there is a $p \in D$ with $n(p)=n(q)$ for all $q \in D$ with $p \leqslant \mathbb{D} q$. 
Proof. Assume, towards a contradiction, that, for every $p \in D$, there is a $q \in D$ with $p \leqslant \mathbb{D} q$ and $n(p)<n(q)$. Then there is a strategy $s:{ }^{<\omega} D \longrightarrow D$ for Player II in the game $\mathcal{G}(\mathbb{D})$ with $n\left(p_{2 i}\right)<n\left(s\left(\left\langle p_{0}, \ldots, p_{2 i}\right\rangle\right)\right)$ for all $i<\omega$ and $p_{0}, \ldots$, $p_{2 i} \in D$. By our assumption, $s$ is not a winning strategy, and there is a run $\left(p_{i}\right)_{i<\omega}$ of $\mathcal{G}(\mathbb{D})$ played according to $s$ that is won by Player I. This gives us a $p \in D$ with $p_{i} \leqslant \mathbb{D} p$ for all $i<\omega$. Then there is an $i<\omega$ with $n\left(p_{i}\right)>n(p)$, which is a contradiction.

We are now ready to show that the map $u_{\mathbb{I}, F}$ defined in (6) can be an isomorphism witnessing that the group $F\left(A_{\mathbb{I}}\right)$ is an inverse limit of $\mathbb{I}_{F}$.

THEOREM 3.10. Let $F:$ Set $\longrightarrow$ Grp be a functor that induces a free construction, and let $\mathbb{I}$ be an inverse system of sets over a directed set $\mathbb{D}$. If Player II has no winning strategy in the game $\mathcal{G}(\mathbb{D})$, then the induced map $u_{\mathbb{I}, F}: F\left(A_{\mathbb{I}}\right) \longrightarrow G_{\mathbb{I}_{F}}$ is an isomorphism, and $F\left(A_{\mathbb{I}}\right)$ is an inverse limit of $\mathbb{I}_{F}$.

Proof. Let $\mathbb{D}=\langle D, \leqslant \mathbb{D}\rangle$ and $\mathbb{I}=\left\langle\left\langle A_{p} \mid p \in D\right\rangle,\left\langle f_{p, q} \mid p \leqslant \mathbb{D} q\right\rangle\right\rangle$. Given sets $\bar{A} \subseteq A$ and $p, q \in D$ with $p \leqslant \mathbb{D} q$, we define $\iota_{\bar{A}, A}=F\left(i_{\bar{A}, A}\right), G_{p}=F\left(A_{p}\right)$ and $h_{p, q}=F\left(f_{p, q}\right)$. If $\emptyset \subsetneq \bar{A} \subseteq A$, then $\iota_{\bar{A}, A}$ has a left inverse. In combination with our assumptions, this shows that all homomorphisms of the form $\iota_{\bar{A}, A}$ are injective.

By Lemma 3.6, it suffices to show that $u_{\mathbb{I}, F}$ is surjective. Fix an element $\left(g_{p}\right)_{p \in D}$ of $G_{\mathbb{I}_{F}}$. Given $p \in D$, let $\bar{A}_{p}$ denote the unique minimal finite subset of $A_{p}$ with $g_{p} \in \operatorname{ran}\left(\iota_{\bar{A}_{p}, A_{p}}\right)$, and set $\bar{G}_{p}=F\left(\bar{A}_{p}\right)$. By the above remarks, there is a unique $\bar{g}_{p} \in \bar{G}_{p}$ with $\iota_{\bar{A}_{p}, A_{p}}\left(\bar{g}_{p}\right)=g_{p}$. Let $n(p)$ denote the cardinality of $\bar{A}_{p}$.

ClAIM 1. If $p \leqslant \mathbb{D} q$, then $n(p) \leqslant n(q)$, and the set $\bar{A}_{p}$ is contained in the image of $\bar{A}_{q}$ under $f_{p, q}$.

Proof of the Claim. We let $\bar{A}$ denote the image of $\bar{A}_{q}$ under $f_{p, q}$, and define $\bar{f}=f_{p, q} \uparrow \bar{A}_{q}: \bar{A}_{q} \longrightarrow \bar{A}$. Then we have that $f_{p, q} \circ i_{\bar{A}_{q}, A_{q}}=i_{\bar{A}, A_{p}} \circ \bar{f}$, and this implies that

$$
g_{p}=h_{p, q}\left(g_{q}\right)=\left(h_{p, q} \circ \iota_{\bar{A}_{q}, A_{q}}\right)\left(\bar{g}_{q}\right)=\left(\iota_{\bar{A}, A_{p}} \circ F(\bar{f})\right)\left(\bar{g}_{q}\right) .
$$

This shows that $g_{p} \in \operatorname{ran}\left(\iota_{\bar{A}, A_{p}}\right), \bar{A}_{p} \subseteq \bar{A}$, and $n(p)=\left|\bar{A}_{p}\right| \leqslant|\bar{A}| \leqslant\left|\bar{A}_{q}\right|=$ $n(q)$.

By Proposition 3.9 and Claim 1, our assumptions imply that there is a $p_{*} \in D$ with $n(q)=n\left(p_{*}\right)$ for all $q \in D$ with $p_{*} \leqslant \mathbb{D} q$. Given $q, r \in D$ with $p_{*} \leqslant \mathbb{D} q \leqslant \mathbb{D}$ $r$, Claim 1 shows that $\bar{A}_{q}$ is equal to the image of $\bar{A}_{r}$ under $f_{q, r}$, the resulting 
function $\bar{f}_{q, r}=f_{q, r} \uparrow \bar{A}_{r}: \bar{A}_{r} \longrightarrow \bar{A}_{q}$ is a bijection, and the map $\bar{h}_{q, r}=F\left(\bar{f}_{q, r}\right)$ : $\bar{G}_{r} \longrightarrow \bar{G}_{q}$ is an isomorphism of groups.

Claim 2. If $q, r \in D$ with $p_{*} \leqslant \mathbb{D} q \leqslant \mathbb{D}$, then $\bar{h}_{q, r}\left(\bar{g}_{r}\right)=\bar{g}_{q}$.

Proof of the Claim. Since $p_{*} \leqslant \mathbb{D} q, r$, we know that $\bar{A}_{q}$ is equal to the image of $\bar{A}_{r}$ under $f_{p, q}$, and this implies that $f_{q, r} \circ i_{\bar{A}_{r}, A_{r}}=i_{\bar{A}_{q}, A_{q}} \circ \bar{f}_{q, r}$. We can conclude that

$$
\iota_{\bar{A}_{q}, A_{q}}\left(\bar{g}_{q}\right)=g_{q}=h_{q, r}\left(g_{r}\right)=\left(h_{q, r} \circ \iota_{\bar{A}_{r}, A_{r}}\right)\left(\bar{g}_{r}\right)=\left(\iota_{\bar{A}_{q}, A_{q}} \circ \bar{h}_{q, r}\right)\left(\bar{g}_{r}\right),
$$

and the injectivity of $\iota_{\bar{A}_{q}, A_{q}}$ yields the statement of the claim.

Claim 3. If $q, r_{0}, r_{1} \in D$ with $q, p_{*} \leqslant \mathbb{D} r_{0}, r_{1}$, then

$$
f_{q, r_{0}} \circ i_{\bar{A}_{r_{0}}, A_{r_{0}}} \circ \bar{f}_{p_{*}, r_{0}}^{-1}=f_{q, r_{1}} \circ i_{\bar{A}_{r_{1}}, A_{r_{1}}} \circ \bar{f}_{p_{*}, r_{1}}^{-1} .
$$

Proof of the Claim. Pick $r \in D$ with $r_{0}, r_{1} \leqslant \mathbb{D} r$ and $i<2$. Then we have that $\bar{f}_{p_{*}, r}=\bar{f}_{p_{*}, r_{i}} \circ \bar{f}_{r_{i}, r}$, and this implies that $\bar{f}_{p_{*}, r_{i}}^{-1}=\bar{f}_{r_{i}, r} \circ \bar{f}_{p_{*}, r}^{-1}$. This shows that

$$
f_{q, r_{i}} \circ i_{\bar{A}_{r_{i}}, A_{r_{i}}} \circ \bar{f}_{p_{*}, r_{i}}^{-1}=f_{q, r_{i}} \circ i_{\bar{A}_{r_{i}}, A_{r_{i}}} \circ \bar{f}_{r_{i}, r} \circ \bar{f}_{p_{*}, r}^{-1}=f_{q, r} \circ i_{\bar{A}_{r}, A_{r}} \circ \bar{f}_{p_{*}, r}^{-1}
$$

holds for all $i<2$.

By the above claim, there is a unique function $f_{*}: \bar{A}_{p_{*}} \longrightarrow A_{\mathbb{I}}$ such that

$$
\mathrm{p}_{q} \circ f_{*}=f_{q, r} \circ i_{\bar{A}_{r}, A_{r}} \circ \bar{f}_{p_{*}, r}^{-1}
$$

holds whenever $q, r \in D$ with $p_{*}, q \leqslant \mathbb{D} r$. Set $h_{*}=F\left(f_{*}\right): \bar{G}_{p_{*}} \longrightarrow F\left(A_{\mathbb{I}}\right)$.

Claim 4. $\left(g_{p}\right)_{p \in D}=\left(u_{\mathbb{I}, F} \circ h_{*}\right)\left(\bar{g}_{p_{*}}\right) \in \operatorname{ran}\left(u_{\mathbb{I}, F}\right)$.

Proof of the Claim. Pick $q \in D$. Then there is an $r \in D$ such that $p_{*}, q \leqslant \mathbb{D} r$ and (7) holds. By Claim 2, we have that

$$
\begin{aligned}
& \left(\pi_{q} \circ u_{\mathbb{I}, F} \circ h_{*}\right)\left(\bar{g}_{p_{*}}\right)=F\left(\mathrm{p}_{q} \circ f_{*}\right)\left(\bar{g}_{p_{*}}\right)=F\left(f_{q, r} \circ i_{\bar{A}_{r}, A_{r}} \circ \bar{f}_{p_{*}, r}^{-1}\right)\left(\bar{g}_{p_{*}}\right) \\
& =\left(h_{q, r} \circ \iota_{\bar{A}_{r}, A_{r}} \circ \bar{h}_{p_{*}, r}^{-1}\right)\left(\bar{g}_{p_{*}}\right)=\left(h_{q, r} \circ \iota_{\bar{A}_{r}, A_{r}}\right)\left(\bar{g}_{r}\right)=h_{q, r}\left(g_{r}\right)=g_{q} .
\end{aligned}
$$

These computations show that $\left(u_{\mathbb{I}, F} \circ h_{*}\right)\left(\bar{g}_{p_{*}}\right)=\left(g_{p}\right)_{p \in D}$.

The above claim completes the proof of the theorem. 


\section{Good inverse systems}

In this section, we complete the proofs of the results listed in Section 1 by the construction of suitable inverse systems of sets from the assumptions appearing in the statements of those results. The next definition makes the notion of suitable inverse system precise.

DEFINITION 4.1. Let $\lambda$ and $v$ be infinite cardinals. We say that an inverse system $\mathbb{I}=\left\langle\left\langle A_{p} \mid p \in D\right\rangle,\left\langle f_{p, q} \mid p \leqslant \mathbb{D} q\right\rangle\right\rangle$ of sets over a directed set $\mathbb{D}=\langle D, \leqslant \mathbb{D}\rangle$ is $(\lambda, v)$-good if the following statements hold.

(1) Player II has no winning strategy in the game $\mathcal{G}(\mathbb{D})$.

(2) $|D| \leqslant \lambda$ and $\left|A_{p}\right| \leqslant \lambda$ for all $p \in D$.

(3) $\left|A_{\mathbb{I}}\right|=v$.

The following corollary summarizes the results of the previous sections.

COROLlary 4.2. Let $F$ : Set $\longrightarrow$ Grp be a functor that induces a free construction with $|F(A)| \leqslant|A|+\aleph_{0}$ for every set A. If there exists a $(\lambda, v)$-good inverse system, then the group $F(v)$ is the automorphism group of a $\lambda$-structure.

Proof. Let $\mathbb{I}_{F}$ denote the corresponding system of groups defined in (5). By our assumptions, we have that $\left|F\left(A_{p}\right)\right| \leqslant \lambda$ for every $p \in D$. In this situation, Theorem 2.4 shows that the inverse limit $G_{\mathbb{I}_{F}}$ of $\mathbb{I}_{F}$ is the automorphism group of a $\lambda$-structure. By Theorem 3.10, the groups $G_{\mathbb{I}_{F}}$ and $F\left(A_{\mathbb{I}}\right)$ are isomorphic. Since our assumptions imply that the groups $F\left(A_{\mathbb{I}}\right)$ and $F(v)$ are also isomorphic, this shows that the group $F(v)$ is the automorphism group of a $\lambda$-structure.

In order to prove Theorem 1.4 , we now construct a $\left(\lambda, 2^{\lambda}\right)$-good inverse system from the assumption that $\lambda=\lambda^{\aleph_{0}}$.

LEMMA 4.3. If $\lambda$ is a cardinal with $\lambda=\lambda^{\aleph_{0}}$, then the inverse system of sets $\mathbb{I}_{\lambda}$ defined in (1) is $\left(\lambda, 2^{\lambda}\right)$-good.

Proof. By our assumption, we have that $\left|[\lambda]^{\aleph_{0}}\right| \leqslant \lambda$ and ||$^{u} 2 \mid \leqslant \lambda$ for all $u \in[\lambda]^{\aleph_{0}}$. Since the function $b$ defined in (2) is a bijection, we have that $\left|A_{\mathbb{I}_{\lambda}}\right|=2^{\lambda}$. Finally, the directed set $\left\langle[\lambda]^{\aleph_{0}}, \subseteq\right\rangle$ is $\sigma$-directed, and an application of Proposition 3.7 shows that Player II has no winning strategy in the corresponding game.

The statement of Theorem 1.4 now follows directly from a combination of Corollary 4.2 and Lemma 4.3. 
Proof of Theorem 1.4. Define $F r:$ Set $\longrightarrow$ Grp to be the functor that sends a set $A$ to the free group with basis $A$. Then $F r$ induces a free construction, and $|\operatorname{Fr}(A)| \leqslant|A|+\aleph_{0}$ holds for every set $A$. Let $\lambda$ be a cardinal with $\lambda=\lambda^{\aleph_{0}}$. By Lemma 4.3 , there exists a $\left(\lambda, 2^{\lambda}\right)$-good inverse system of sets. In this situation, Corollary 4.2 shows that the group $\operatorname{Fr}\left(2^{\lambda}\right)$ is the automorphism group of a $\lambda$-structure.

Next, we show that Corollary 1.5 is a direct consequence of Theorem 1.4 and the results presented in the first two sections.

Proof of Corollary 1.5. Assume that the Continuum Hypothesis and the Singular Cardinal Hypothesis hold. Let $\lambda$ be an infinite cardinal.

If $\operatorname{cof}(\lambda)>\omega$, then our assumptions and [8, Theorem 5.22.(ii).(b)] imply that $\lambda=\lambda^{\aleph_{0}}$, and in this situation Theorem 1.4 shows that the free group of rank $2^{\lambda}$ is the automorphism group of a $\lambda$-structure.

Next, assume that $\operatorname{cof}(\lambda)=\omega$ and that there is a cardinal $\kappa<\lambda$ with $2^{\kappa}>\lambda$. Then we can find a regular uncountable cardinal $\kappa$ with these properties, and the above argument shows that the free group of rank $2^{\kappa}$ is the automorphism group of a $\kappa$-structure. Hence this group is also the automorphism group of a $\lambda$-structure.

Finally, assume that $\operatorname{cof}(\lambda)=\omega$ and $2^{\kappa} \leqslant \lambda$ holds for all $\kappa<\lambda$. Then either $\lambda=\aleph_{0}$ or $\lambda$ is a singular strong limit cardinal of countable cofinality. In this situation, Theorem 1.2 and Theorem 1.3 imply that there is no free group of rank greater than $\lambda$ that is the automorphism group of a $\lambda$-structure.

Next, we prove Theorem 1.6. The following notion was introduced by Hamkins (see [5]).

DEFINITION 4.4. Let $M$ be an inner model of ZFC. We say that $M$ has the $\omega_{1}$-cover property if every countable set of ordinals in $\mathrm{V}$ is a subset of a set that is an element of $M$ and countable in $M$.

LEMMA 4.5. Let $M$ be an inner model of ZFC with the $\omega_{1}$-cover property. If $\lambda$ is an infinite cardinal such that $\lambda=\left(\lambda^{\aleph_{0}}\right)^{M}$ and $\left(2^{\lambda}\right)^{M}$ is a cardinal in $\mathrm{V}$, then there is a $(\lambda, v)$-good inverse system of sets for some cardinal $v \geqslant\left(2^{\lambda}\right)^{M}$.

Proof. Let $\mathbb{I}=\mathbb{I}_{\lambda}^{M}$ be the inverse system of sets defined by (1) in $M$, and let $v$ be the cardinality of $A_{\mathbb{I}}$ in $\mathrm{V}$. Since every element of $\left({ }^{\lambda} 2\right)^{M}$ gives rise to a distinct element of $A_{\mathbb{I}}$ and $\left(2^{\lambda}\right)^{M}$ is a cardinal in $\mathrm{V}$, we have that $v \geqslant\left(2^{\lambda}\right)^{M}$. By Proposition 3.7, our assumption implies that the directed set $\left\langle\left([\lambda]^{\aleph_{0}}\right)^{M}, \subseteq\right\rangle$ is $\sigma$-directed in $\mathrm{V}$, and hence Player II has no winning strategy in the corresponding game. We can conclude that the inverse system $\mathbb{I}$ is $(\lambda, \nu)$-good in $\mathrm{V}$. 
Proof of Theorem 1.6. Let $\lambda$ be a cardinal with $\lambda=\lambda^{\aleph_{0}}$, and let $G$ be $\operatorname{Add}(\omega, \kappa)$ generic over the ground model V for some cardinal $\kappa$. Since $\operatorname{Add}(\omega, \kappa)$ satisfies the countable chain condition, the ground model $\mathrm{V}$ has the $\omega_{1}$-cover property in $\mathrm{V}[G]$, and $\left(2^{\lambda}\right)^{\mathrm{V}}$ is still a cardinal in $\mathrm{V}[G]$. Now, the statement of the theorem directly follows from Corollary 4.2 and Lemma 4.5.

Finally, the results of the last two section also allow us to prove Theorem 1.7.

Proof of Theorem 1.7. Let $\lambda$ be a cardinal of uncountable cofinality, and let $\mathbb{T}$ be a tree of cardinality and height $\lambda$ with the property that the set [ $\mathbb{T}]$ of branches through $\mathbb{T}$ of order-type $\lambda$ has infinite cardinality $v>\lambda$. By Proposition 3.7, the assumption that $\operatorname{cof}(\lambda)>\omega$ implies that the directed set $\langle\lambda, \leqslant\rangle$ is $\sigma$-directed, and hence Player II has no winning strategy in the corresponding game. Since the computations in Example 3.3 show that the inverse limit of the inverse system $\mathbb{I}_{\mathbb{T}}$ defined in (3) also has cardinality $v$, we can conclude that $\mathbb{I}_{\mathbb{T}}$ is $(\lambda, v)$-good, and Corollary 4.2 yields the statement of the theorem.

\section{Open questions}

We close this paper with questions raised by the above results.

QUESTION 5.1. Is it consistent with the axioms of ZFC that there is a cardinal $\lambda$ of uncountable cofinality with the property that the free group of rank $2^{\lambda}$ is not the automorphism group of a $\lambda$-structure?

QUESTION 5.2. Is it consistent with the axioms of ZFC that there is a cardinal $\lambda$ of uncountable cofinality with the property that every free group of rank greater than $\lambda$ is not the automorphism group of a $\lambda$-structure?

QUESTION 5.3. Is it consistent with the axioms of ZFC that there is a singular cardinal $\lambda$ of uncountable cofinality with the property that there is no tree of cardinality and height $\lambda$ with more than $\lambda$-many branches of order-type $\lambda$ ?

\section{Acknowledgements}

The second author would like to thank the Israel Science Foundation for partial support of this research (Grant No. 1053/11). Both authors would like to thank the two anonymous referees for their careful reading of the paper, their corrections, and numerous useful suggestions. 


\section{References}

[1] J. E. Baumgartner, 'Applications of the proper forcing axiom', in Handbook of set-theoretic topology (North-Holland, Amsterdam, 1984), 913-959.

[2] J. E. Baumgartner, 'Iterated forcing', in Surveys in Set Theory, London Math. Soc. Lecture Note Ser., 87 (Cambridge Univ. Press, Cambridge, 1983), 1-59.

[3] N. G. de Bruijn, 'Embedding theorems for infinite groups', Nederl. Akad. Wetensch. Proc. Ser. A. 60 = Indag. Math 19 (1957), 560-569.

[4] E. Fried and J. Kollár, 'Automorphism groups of fields', in Universal Algebra (Esztergom, 1977), Colloq. Math. Soc. János Bolyai, 29 (North-Holland, Amsterdam, 1982), 293-303.

[5] J. D. Hamkins, 'Extensions with the approximation and cover properties have no new large cardinals', Fund. Math. 180 (3) (2003), 257-277.

[6] W. Hodges, Model Theory, Encyclopedia of Mathematics and its Applications, 42 (Cambridge University Press, Cambridge, 1993).

[7] T. Jech, 'More game-theoretic properties of Boolean algebras', Ann. Pure Appl. Logic. 26 (1) (1984), 11-29.

[8] T. Jech, Set Theory, Springer Monographs in Mathematics (Springer-Verlag, Berlin, 2003), The third millennium edition, revised and expanded.

[9] R. Jensen and J. Steel, 'K without the measurable', J. Symbolic Logic. 78 (3) (2013), 708-734.

[10] W. Just, S. Shelah and S. Thomas, 'The automorphism tower problem revisited', Adv. Math. 148 (2) (1999), 243-265.

[11] I. Kaplan and S. Shelah, 'Automorphism towers and automorphism groups of fields without choice', in Groups and Model Theory, Contemp. Math, 576 (Amer. Math. Soc., Providence, RI, 2012), 187-203.

[12] S. Mac Lane, Categories for the Working Mathematician, 2nd edn, Graduate Texts in Mathematics, 5 (Springer-Verlag, New York, 1998).

[13] W. Mitchell, 'Aronszajn trees and the independence of the transfer property', Ann. Math. Logic. 5 (1972/73), 21-46.

[14] R. Sanerib, 'Automorphism groups of ultrafilters', Algebra Universalis. 4 (1974), 141-150.

[15] E. Schimmerling, 'A core model toolbox and guide', in Handbook of Set Theory Vols. 1, 2, 3 (Springer, Dordrecht, 2010), 1685-1751.

[16] S. Shelah, 'Applications of PCF theory', J. Symbolic Logic. 65 (4) (2000), 1624-1674.

[17] S. Shelah, 'A countable structure does not have a free uncountable automorphism group', Bull. London Math. Soc. 35 (1) (2003), 1-7.

[18] S. Shelah, Cardinal arithmetic, Oxford Logic Guides, 29 (Oxford University Press, New York, 1994), Oxford Science Publications.

[19] S. Solecki, 'Polish group topologies', in Sets and Proofs Leeds, 1997, London Math. Soc. Lecture Note Ser., 258 (Cambridge University Press, Cambridge, 1999), 339-364. 